\title{
Carotid Body Tumor - Case Report and Review of the Literature
}

\section{Lezrag $\mathrm{M}^{*}$, Aboulfadl $\mathrm{M}^{2}$, Baghdadi $\mathrm{T}^{3}$, Abada $\mathrm{R}^{1}$, Rouadi $\mathrm{S}^{1}$, Roubal $\mathrm{M}^{1}$ and Mahtar $\mathrm{M}^{1}$}

${ }^{1}$ Department of ENT, 20 August Hospital, Ibn Rochd University Hospital, Casablanca, Morocco

${ }^{2}$ Faculty of Medicine and Pharmacy, Casablanca, Morocco

${ }^{3}$ Department of Surgery, Ibn Rushd Chu, Casablanca, Morocco

${ }^{*}$ Corresponding author: Lezrag M, Department of ENT, 20 August Hospital, Ibn Rochd University Hospital, Casablanca, Morocco, E-mail: lezragmokamed13@gmail.com

Citation: Lezrag M, Aboulfadl M, Baghdadi T, Abada R, Rouadi S, et al. (2016) Carotid Body Tumor - Case Report and Review of the Literature. J Case Rep Stud 4(4): 406. doi: 10.15744/2348-9820.4.406

Received Date: April 21, 2016 Accepted Date: August 29, 2016 Published Date: August 31, 2016

\begin{abstract}
The paraganglioma of the carotid sinus is a relatively rare tumor, which represents the majority of paraganglioma of the head and neck (60-70\%). We report the case of a female patient of 40 years who consulted for a swelling laterocervical high right without associated clinical signs. Angiography and cervicofacial scan had confirmed the vascular nature of the lesion and suspected diagnosis of carotid paraganglioma. Surgical excision was confirmed the diagnosis on histopathologic examination. From this observation, we review the clinical, radiological and histopathological features of the tumor and its uncertain evolutionary mode and therapeutic modalities.

Keywords: Paraganglioma; Chemodectoma; Carotid
\end{abstract}

\section{Introduction}

Paragangliomas are neuroendocrine neoplasm in the Autonomic Nervous System, $90 \%$ arise from the adrenals (pheochromocytoma), $8.5 \%$ in abdomen and $0.3 \%$ found in the head and neck.

Paraganglioma arising from the carotid body are relatively rare tumors but constitute majority of head and neck paragangliomas $(60-70 \%)[1,2]$. The term paraganglia was first used by Kohn in the early twentieth century and is the most appropriate nomenclature from an embryologic standpoint [3-5].

\section{Case Presentation}

A 40-years-old white female patient, presented to the head and neck service in 20 august hospital in Casablanca, Morocco with a history of a painless growing mass on the right side of the neck during the preceding seven years.

The examination found anorexia and an evening heat without sweating without deterioration of general condition.

Examination showing $3 \mathrm{~cm} \times 3 \mathrm{~cm}$ swelling just beneath the sternocleidomastoid that was well circumscribed, pulsatile and smooth. Computer tomography revealed a $5 / 3 / 2.5 \mathrm{~cm}$, mass on the bifurcation of the right common carotid artery.

Magnetic Resonance Angiography (MRA) showed a well-defined tumor of $45 * 30 * 25$ mm discreetly heterogeneous hypointense in T1, hyperintense in FLAIR and DPT2 and taking the contrast, with a partial aspect of salt and pepper (Figure 1).

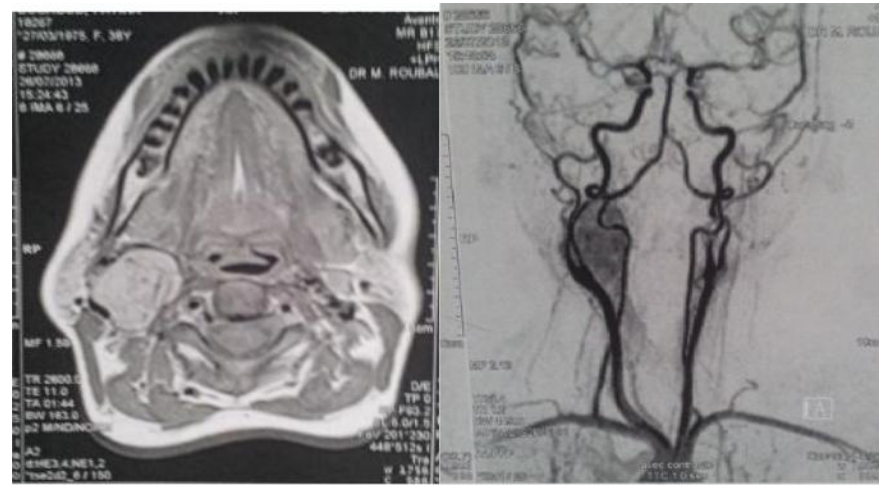

Figure 1: Magnetic resonance angiogram showing a well-defined tumor arising from the region of the external carotid artery (left), Arteriography demonstrated a hypervascularized mass occupying and deforming the right-side carotid bifurcation (right) 
Angiography showed a well-defined tumor arising from the region of the external carotid artery with branches of the ECA as feeders vessels such as posterior inferior cerebellar artery (PICA), and an inferior cerebellar artery (AICA).

The tumor was diagnosed as carotid body paraganglioma of Shamblin group I.

The patient was informed of the high risk of the surgery; consent was obtained.

The patient was not submitted to tumor embolization previously to the surgical excision.

The tumor was approached through a vertical incision over the sternomastoid. subadventitial tumor, the Internal carotid artery was preserved, we did not performed a bypass with prosthetic graft or safenous vein and all cranial nerves were preserved (Figure $2)$.

Excision was performed and sent for histopathological examination.

The histological examination showed a Proliferation arranged in lobules of polygonal cells with granulous cytoplasm with no signs of malignancy.

There was no evidence of neurological deficit after the surgery.

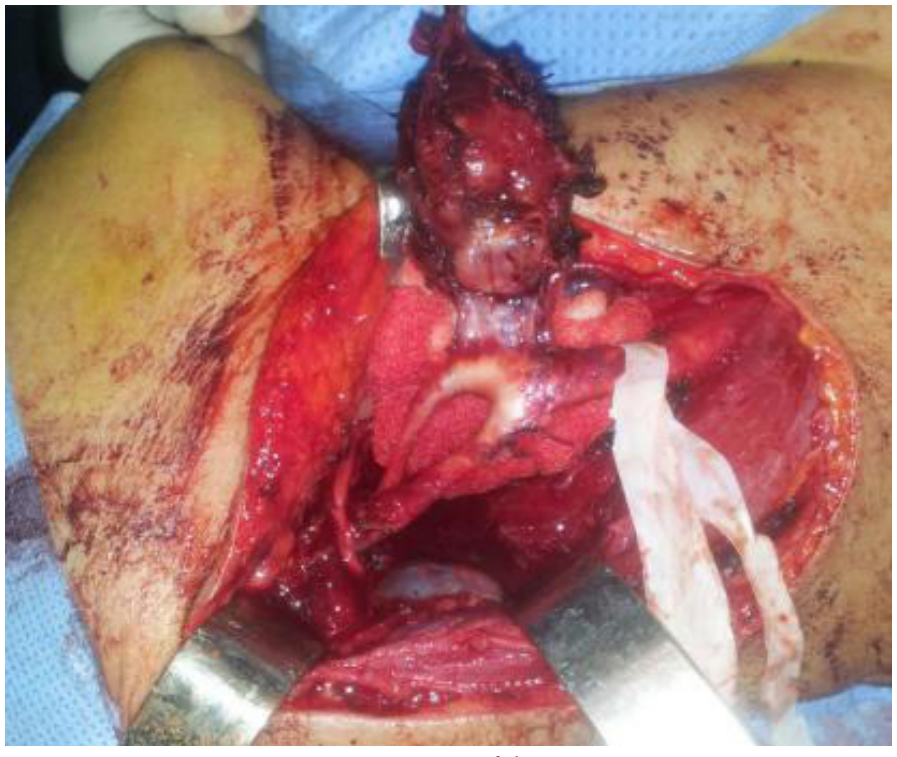

Figure 2: Excision of the tumour

The patient was followed-up at 6 months and every year after for with no local, regional or distant metastasis noticed.

\section{Discussion}

Paragangliomas of the head and neck are highly vascular neoplasms. They most commonly occur at the carotid bifurcation, where they are referred to as carotid body tumors. Other common sites of origin are the jugular bulb (jugular paraganglioma), the tympanic plexus on the promontory (tympanic paraganglioma) and the vagal nerve (vagal paraganglioma) [4,5].

The interest of the carotid body tumor lay in the facts that it is the most common paraganglioma in the head and neck and seems to occur slightly more often in women and more frequently in people living at high altitudes.

Its sporadic form is the most common one and about $5 \%$ of the cases are bilateral. And its familial form, is rarer with a pattern of autosomal, dominant inheritance, and about $32 \%$ of bilateral cases.

Malignancy occurs in 6-12.5\% of cases, which ranks carotid body paragangliomas as the most frequently occurring malignant head and neck paraganglioma $[4,5]$.

Carotid bodies are ellipsoid, red-brown formations, 5 to $7 \mathrm{~mm}$ in height by 2.5 to $4 \mathrm{~mm}$ in width, located in the posterior face of the common carotid artery bifurcation (as described by Von Haller in 1743). On gross examination the tumors are usually wellcircumscribed. The actual size of the tumors may vary greatly [2].

The function of the carotid body is related to autonomic control of the respiratory and cardiovascular systems, as well as blood temperature [1].

Because of its location in close approximation to carotid vessels and X-XII cranial nerves, tumors enlargement causes progressive symptoms such as dysphagia, odynophagia, hoarseness of voice or other cranial nerve deficits but in general Carotid body tumors are typically painless, slow growing tumors that are often present for years prior to the patient seeking medical attention which explain the large sizes found in literature Some have reported tumors as large as $10 \mathrm{~cm} \mathrm{[3].}$ 
The patients may give a history suggestive of symptoms associated with catecholamine production such as fluctuating hypertension, blushing, obstructive sleep apnea and palpitations.

The differential diagnosis includes lymphadenopathies, bronchial cleft cysts, salivary gland tumors, neurogenic tumors, aneurysms of the carotid artery as well as HNPs [5]. In addition, other neuroendocrine tumors such as medullary thyroid carcinoma and neuroendocrine carcinoma should also be included in the differential diagnosis.

Resection of carotid body paraganglioma carries inherent risks of injury to the cranial nerves, carotid arteries as well excessive blood loss [4].

The surgical excision with careful subadventitial dissection is treatment of choice for most carotid body paragangliomas, concerning the bleeding, clamping of all carotid arteries is useful, and with placement of internal carotid shunt some other articles recommend angiographic embolization preoperatively. The Preoperative embolization of a carotid body paraganglioma can be performed by ethanol or polyvinyl alcohol. The finally result is a complete devascularization $[4,5]$.

Overall prognosis is quite good with complete surgical resection. Continued follow-up is necessary, however, as recurrence and metastasis may occur years later [5].

\section{Conclusion}

The carotid body paraganglioma is a rare vascular tumor of the neck that is diagnosed by imaging and not by biopsy. Surgical excision is advised after obtaining a good road map using angiography or MRI. Preoperative functional assessment is essential to lessen perioperative risks.

\section{References}

1. Meyer FB, Sundt TM, Pearson BW (1986) Carotid body tumors: a subject review and suggested surgical approach. J Neurosurg 64: $377-85$.

2. Luna-Ortiz K, Rascon-Ortiz M, Villavicencio-Valencia V, Granados-Garcia M, Herrera-Gomez A (2005) Carotid body tumors: Review of a 20-year experience. Oral Oncol 41: 56-61.

3. Erickson D, Kudva YC, Ebersold MJ, Thompson GB, Grant CS, et al. (2001) Benign paragangliomas: Clinical presentation and treatment outcomes in 236 patients. J Clin Endocrinol Metab 86: 5210-6.

4. Horowitz M, Whisnant RE, Jungreis C, Snyderman C, Levy EI, et al. (2002) Temporary balloon occlusion and ethanol injection for preoperative embolization of carotid-body tumor. Ear Nose Throat J 81: 536-8.

5. Hallett JW, Nora JD, Hollier LH, Cherry KJ, Pairolero PC (1988) Trends in neurovascular complications of surgical management for carotid body and cervical paragangliomas: a fifty-year experience with 153 tumors. J Vasc Surg 7: 284-91.

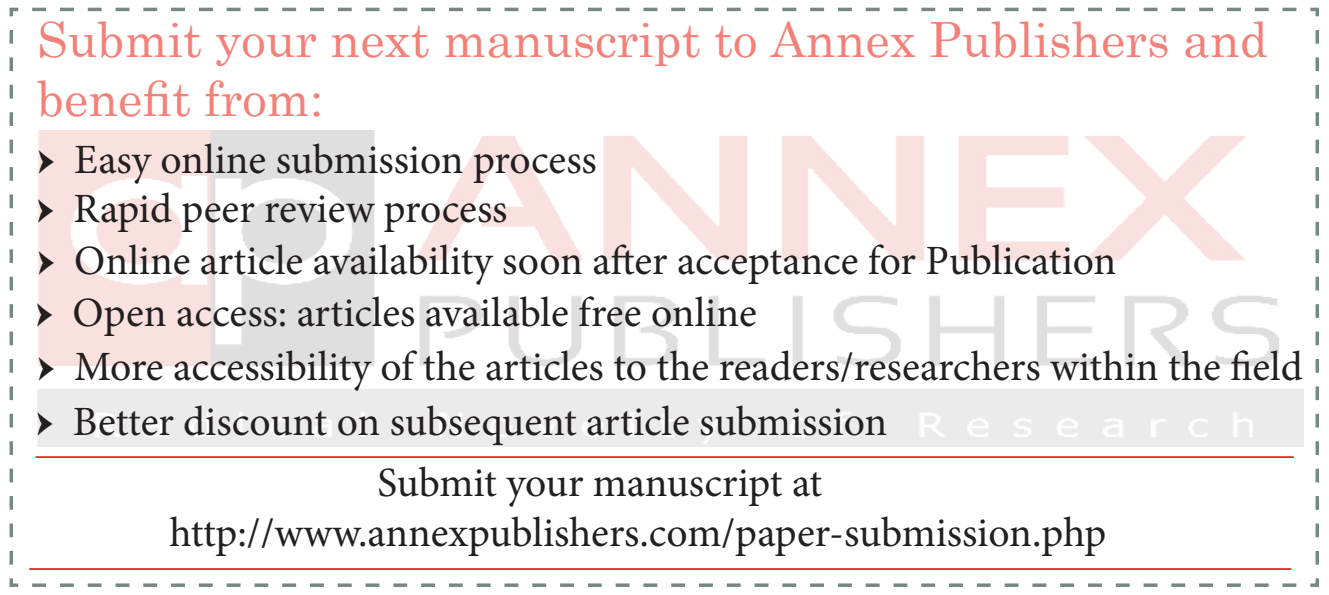

